Hansjörg Donnerberg

\title{
Atomic Simulation \\ of Electrooptic and Magnetooptic Oxide Materials
}

With 45 Figures and 40 Tables

Springer 


\section{Contents}

1. Introduction $\ldots \ldots \ldots \ldots \ldots \ldots \ldots, \ldots \ldots \ldots, \ldots, \ldots, 1$

2. The Scope of Theoretical Methods............... 7

2.1 Electronic Structure Calculations ................... 9

2.1.1 Perfect Crystals ........................... 15

2.1.2 Defective Crystals ........................ 18

2.2 Potential Simulations $\ldots \ldots \ldots \ldots \ldots \ldots \ldots \ldots \ldots \ldots, 33$

2.2.1 Potential Forms and Applications $\ldots \ldots \ldots \ldots \ldots \ldots . . . .33$

2.2.2 Derivation of Effective Crystal Potentials ......... 40

3. Barium Titanate........................... 49

3.1 Ferroelectricity of $\mathrm{ABO}_{3}$ Perovskites .............. 51

3.2 Defect Chemical Properties of Barium Titanate .......... 54

3.3 Electronic Structure Calculations ................. 58

3.3.1 Embedded Cluster Calculations

for Transition Metal Ions in $\mathrm{BaTiO}_{3} \ldots \ldots \ldots \ldots \ldots, 61$

3.3.2 Simulation of Trapped Holes ............... 77

3.3.3 Simulation of Electron-Type Bipolarons ......... 97

3.3.4 Embedded Cluster Calculations of Oxygen Vacancies in $\mathrm{BaTiO}_{3} \ldots \ldots \ldots \ldots \ldots \ldots \ldots \ldots \ldots, \ldots$

4. Potassium Niobate and Potassium Tantalate .......... 105

4.1 Shell Model Simulations in $\mathrm{KNbO}_{3}$ and $\mathrm{KTaO}_{3} \ldots \ldots \ldots \ldots 106$

4.1.1 Shell Model Parameters

and Perfect Lattice Simulations ............... 106

4.1.2 Defect Chemistry of $\mathrm{KTaO}_{3}$ and $\mathrm{KNbO}_{3} \ldots \ldots \ldots \ldots 114$

4.2 Polarizing Defect Centres ...................... 119

4.2.1 Dipole Formation Due to Off-Centre Displacements of Defects .......................... 122

4.2.2 Dipole Centres Due to Defect Aggregation.......... 132

5. Lithium Niobate $\ldots \ldots \ldots \ldots \ldots \ldots \ldots \ldots \ldots \ldots \ldots \ldots \ldots \ldots$

5.1 Electronic Structure Calculations ................. 147

5.2 Shell Model Simulations of Defect Chemical Properties ...... 150 
VIII Contents

5.2 .1 Intrinsic Defect Structure.............. 150

5.2 .2 Incorporation of Impurities . . . . . . . . . . 154

6. Strontium Barium Niobate $\ldots \ldots \ldots \ldots \ldots \ldots \ldots \ldots \ldots \ldots \ldots \ldots$

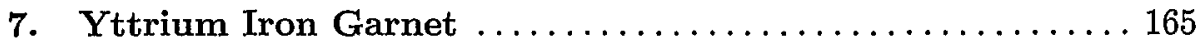

7.1 Potential Models . . . . . . . . . . . . . . . . . . . 165

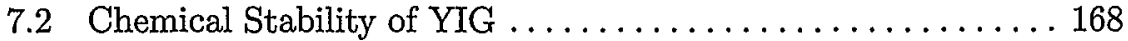

7.3 Intrinsic Defect Structures . . . . . . . . . . . . . . 170

7.4 Intrinsic Electronic Properties . . . . . . . . . . . . 173

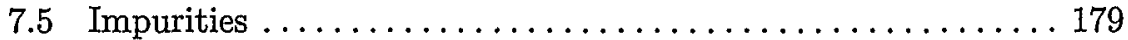

7.5.1 Incorporation of Trivalent Impurities............ 181

7.5.2 Incorporation of Divalent and Tetravalent Impurities . . 184

8. Summary and Conclusions .................. 191

References.............................. 193

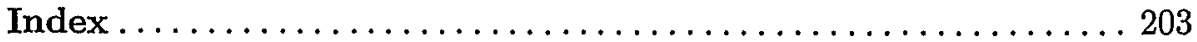

